\title{
THE DISTRIBUTION OF MODULAR REPRESENTATIONS INTO BLOCKS
}

\author{
DAVID W. BURRY
}

\begin{abstract}
The $p$-modular representations of a finite group that are induced from a $p$-subgroup are investigated. A series of three results describing how these representations are distributed into $p$-blocks are presented. Several applications are discussed, including the result that there are a finite number of indecomposable $p$-modular representations (up to equivalence) in a $p$-block of a group if and only if its defect group is cyclic.
\end{abstract}

The notation and point of view will be that of Dornhoff [2]. Fix a finite group $G$, a prime integer $p$, and a characteristic $p$ field $F$. All modules are assumed to be finitely generated.

The author wishes to thank Professor L. L. Scott for his contributions to the development of the results in this article.

TheOREM 1. Suppose $P$ is a p-subgroup of $G$, and $L$ is an irreducible $F G$-module. Then there is a positive integer $n=n(P, L)$ with the property that for any $F P$-module $M, M^{G}$ has $L$ as a composition factor $n(\operatorname{dim} M)$ times.

Proof. Let $M=M_{n} \supset \cdots \supset M_{1} \supset M_{0}=\{0\}$ be a composition series of $F P$-modules. Since $P$ is a $p$-group, each factor is isomorphic to $T$, the trivial $F P$-module. Then $M^{G}=M_{n}^{G} \supset \cdots \supset M_{1}^{G} \supset M_{0}^{G}=\{0\}$ is a series of $F G$ modules with each factor isomorphic to $T^{G}$. This gives the theorem except for the possibility of $n$ being zero. To show that $n$ is positive, it is enough to show $L$ is a composition factor of $T^{G}$. Again since $P$ is a $p$-group, $\operatorname{Hom}_{P}\left(L_{P}, T\right) \neq$ $\{0\}$. Thus by Frobenius reciprocity (see [5, Remark 2 in 7.1]),

$$
0 \neq \operatorname{dim}\left(\operatorname{Hom}_{P}\left(L_{P}, T\right)\right)=\operatorname{dim}\left(\operatorname{Hom}_{G}\left(L, T^{G}\right)\right) .
$$

Hence $L$ is a composition factor of $T^{G}$, as required.

Theorem 2. Suppose $P$ is a p-subgroup of $G$, and $M$ is an FP-module. Then $M^{G}$ has direct summands in every $R G$-block.

Proof. By Theorem 1, every irreducible $F G$-module is a composition factor of $M^{G}$. Hence by the structure of the Cartan matrix (see $[2,46.2]$ ), the theorem holds.

Received by the editors January 24, 1979.

AMS (MOS) subject classifications (1970). Primary 20C05, $20 \mathrm{C20}$.

Key words and phrases. Induced module, block, defect group, source.

(C) 1980 American Mathematical Society 0002-9939/80/0000-0004/\$01.75 
REMARKS. (1) For groups of Lie type, forms of the above theorems are known to specialists. (2) Theorems 1 and 2 give constraints on the dimension of a direct summand of a module induced from a $p$-subgroup in terms of the number of $F G$-blocks and the dimensions of the irreducibles in them. (3) If $F G$ has a projective irreducible module, by Theorem 2 , it must be a direct summand of every module induced from a $p$-subgroup.

THEOREM 3. Suppose $B$ is an FG-block, and $Q$ is a subgroup of a defect group of $B$. Then for any $F Q$-module $S$ that is a source, there is some $F G$-module in $B$ with source $S$.

Proof. Since $Q$ is inside a defect group of $B$, there is an $H=Q C_{G}(Q)$ block $b$ with $b^{G}=B$ (see Lemma 4 below). Applying Theorem 2 with $H$ in the role of $G$, there is an indecomposable $F H$-module $U$ with $U \mid S^{H}$ and $U \in b$. Since $\left(S^{H}\right)_{Q}$ is a direct sum of copies of $S, U$ must have source $S$. From source theory, there is an $F G$-module $V$ with source $S$ and $U \mid V_{H}$ (see [1, Theorem 11]). Since $U$ and $V$ have the same vertex and $U \mid V_{H}$, Nagao's theorem tells us that they are in blocks related by the Brauer correspondence (see $[2,56.5]$ ). In other words, $V \in b^{G}=B$; so we are done.

Higman [4] proved that there are a finite number of indecomposable $F G$-modules (up to isomorphism) if and only if the $p$-Sylow subgroups of $G$ are cyclic. Roughly, his proof developed the theory of sources and then noted there are a finite number of sources over a particular $p$-subgroup if and only if it is cyclic. Theorem 3 represents the natural refinement of Higman's work to $p$-blocks, giving that there are a finite number of indecomposable $F G$-modules (up to isomorphism) in a $F G$-block if and only if its defect group is cyclic. Hamernik [3] has obtained the result just stated, but nothing as strong as Theorem 3. Also his technique requires more sophisticated tools such as the Green correspondence.

No direct statement and elementary proof of Lemma 4 appears in the literature, in spite of its being well known. Thus, for the convenience of the reader, a proof is supplied.

LemMA 4. Suppose $Q$ is a p-subgroup of $G$. Then the Brauer correspondence sends all $Q C_{G}(Q)$-blocks onto all FG-blocks with defect group containing $Q$.

Proof. From [2, 57.4 and 54.11], the Brauer correspondence sends all $H=Q C_{G}(Q)$-blocks into all $F G$-blocks with defect group containing $Q$. Let $B$ be an $F G$-block with defect group $D$ containing $Q$. We need an $F H$-block $b$ with $b^{G}=B$. Let $e$ be the centrally primitive idempotent of $B$. We need $s(e) \neq 0$, where $s$ is the Brauer homomorphism. By the definition of defect group, there is a conjugacy class $C$ of $G$ with defect group $D$ such that the coefficient of $\hat{C}$ in the expression of $e$ is not zero. Since $C$ has defect group $D$, it has elements centralizing $D$, and consequently $Q$. Hence $C \cap C_{G}(Q) \neq \varnothing$. Thus $s(e)$ has nonzero coefficients for all $H$-class sums coming from $C \cap$ $C_{G}(Q)$. 


\section{REFERENCES}

1. D. W. Burry, A strengthened theory of vertices and sources, J. Algebra (to appear).

2. L. Dornhoff, Group representation theory, Pure and Appl. Math., vol. 7, Dekker, New York, 1972.

3. W. Hamernik, Indecomposable modules with cyclic vertix, Math. Z. 142 (1975), 87-90.

4. D. G. Higman, Indecomposable representations at characteristic p, Duke Math. J. 21 (1954), 377-381.

5. J.-P. Serre, Linear representations of finite groups, Graduate Texts in Math., vol. 42, Springer-Verlag, Berlin and New York, 1977.

Department of Mathematics, Yale University, New Haven, Connecticut 06520 